\title{
Some aspects of simplified modeling of tokamak plasmas in a computational electromagnetic environment
}

\author{
J. Sakellaris \\ National Technical University of Athens, Greece
}

\begin{abstract}
Next to the experimental approach of phenomena concerning plasma and plasma facing components of a tokamak another approach was adopted in this paper, a numerical modelling one. The study was done using CARIDDI, which is a 3D integral code for the eddy current density $\mathbf{J}$, the problem is solved by computing at every edge of the finite element mesh the projection of a vector, the current vector potential $\mathbf{T}$, whose uniqueness can be demonstrated mathematically by appropriately using the notions of tree and co-tree formed by branches of a graph, according to graph theory. A plasma is considered to be a toroidal conductor with an elliptical or rectangular cross-section. The section is subdivided into elements and the plasma current is carried in each single element by a filament, placed in its center.
\end{abstract}

Keywords: modeling, tokamak, plasma equilibria, computational electromagnetic environment.

\section{Introduction}

Controlled Thermonuclear Fusion technology has advanced rapidly during the last few years.

The progress in the design and R\&D activity undertaken in the Russian Federation in the period after completion of the ITER EDA is considerable. For the last 3 years, since 2001 FDR, the ITER design has been evolved in detail to resolve issues, to reduce costs, to define details and to prepare the procurement specifications for long-term items. During the ITER Transitional Arrangements (ITA) phase (2003-2004) the Design and R\&D activity in the Russian Federation has been redirected and mainly concentrated on items in accordance 
with the prospective procurement allocation. During the last 2 years, 33 Task Agreements were allocated to the Russian Federation Participant Team (RF PT). The list of Tasks includes the qualification of $\mathrm{Nb}_{3} \mathrm{Sn}$ strands, manufacturing of NbTi cable for the PF (poloidal field) insert coil, qualification tasks for the FW (firstb wall) and PFC/Divertor and design and manufacturing studies related to various areas: magnets, vacuum vessel, blanket, divertor, plasma-facing components, assembly, thermal shield, cryoplant, nuclear analysis, materials and safety [1].

The experimental study of liquid metals (Ga, Li) as tokamak Plasma Facing Component (PFC) was undertaken in Russian T-3M and T-11M tokamaks $\left(\mathrm{I}_{\mathrm{p}} \leq 100 \mathrm{k} \mathrm{A}, \mathrm{B}_{\mathrm{T}} \approx 1 \mathrm{~T}\right)$. In the $\mathrm{T}-3 \mathrm{M}$ droplet stream and film flow Ga limiters were tested. In $\mathrm{T}-11 \mathrm{M}$ the experiments with Li capillary pore systems (CPS) as a rail limiter for investigation of real Li losses in tokamak boundary conditions were performed. It was shown that a liquid metal (Ga, Li) PFC can be used in a tokamak as droplet and CPS structures. The main channel of lithium erosion looks like ion sputtering. The motion towards the tokamak reactor with Li PFC seems possible and has no serious physical obstacles [2].

The morphology of carbon plasma facing components retrieved from the TEXTOR tokamak after long operation periods and exposure to total particle doses exceeding $7 \times 10^{26} \mathrm{~m}^{-2}$ was determined too. Emphasis was on the composition and structure of the erosion zones. Tiles from two limiters- the main toroidal belt pump ALT-II and auxiliary inner bumper-were examined using high-resolution microscopy, surface profilometry, ion beam analysis techniques and energy dispersive X-ray spectroscopy. The essence of results regarding the net-erosion zones as follows: (i) microstructure of surfaces is significantly smoother than on a non-exposed graphite, whereas carbon fibre composites show similar appearance prior to the exposure and after; (ii) deuterium retention is $2-5 \times 10^{21} \mathrm{~m}^{-2}$; (iii) the presence of plasma impurity atoms (e.g. metals) is detected predominantly in small cavities acting as local shadowed areas on the surface. The results are discussed in terms of processes of material erosion/re-deposition and tokamak operation conditions influencing the morphology of wall components [3].

On the other hand, the development, design, manufacture and testing of actively cooled high heat flux (HHF) plasma facing components (PFCs) has been an essential part of the Tore Supra programme towards long powerful tokamak operation. The Tore Supra PFC programme has culminated in the installation and operation of a toroidal pump limiter, since 2002, which has already allowed it to reach new world records in steady state operation (1 GJ injected in a 6 min discharge). The HHF PFCs development and manufacturing was achieved through a long lead development and industrialisation programme (about 10 years) marked out with a number of challenges. The major technical topics cope with bonding technology analysis involving an adequate material selection and procurement, repair processes development and implementation, development of destructive and non-destructive testing methods, and more generally industrialisation assessment. All these lessons are relevant to the ITER divertor PFCs manufacturing, although the technical solution adopted for Tore Supra (flat 
tiles concept) is different from that proposed for the ITER divertor (monoblock concept).

The routine operation of the actively cooled toroidal pumped limiter (TPL), capable of sustaining up to $10 \mathrm{MW} \mathrm{m}^{-2}$ of nominal convected heat flux, was obtained. Up to now, the limiter of Tore Supra fulfills its objectives in terms of heat exhaust. However, the thermographic monitoring exhibits unexpected behaviour of the surface temperature. Particle exhaust control displays a complex pattern, due to the high fraction of the injected deuterium, which remains in the wall. The first experimental results with a full actively cooled wall give access to ITER relevant information on wall conditioning, hydrogen plasma density and vacuum vessel inventory control, carbon erosion and redeposition and capability of in situ monitoring in a completely actively cooled environment [4].

Last but not least, the Mega Amp Spherical Tokamak (MAST) at Culham is one of the leading world machines studying the spherical tokamak (ST) concept. At the time of the initial construction in 1998 little was known about the sort of divertor structures that would be required in an ST. The machine was therefore provided with relatively rudimentary structures that were designed mostly to protect important components from the hot plasma. While these have served the machine well it was accepted that they might not be suitable when operating MAST to its full potential.

The years of experience of operating MAST have led to the design, manufacture and now installation of a new divertor, the MAST improved divertor (MID) that should be able to cope with the full performance of the machine. The design is based on imbricated (fan-shaped) disks of tiles at the top and bottom of the machine for the outer strike points, giving an excellent compromise between power handling and diagnostic access, with substantial new centre column strike point armour and a shaped plate in between. High purity graphite is chosen as the plasma facing material in preference to CFC since in this case it has a better balance of performance and cost. The lower imbricated disk is insulated in alternate sectors for studies of divertor biasing and extensive diagnostics and additional inboard gas injection are included [5].

\section{Models describing plasma behavior in a tokamak device}

Sophisticated computer modeling must be undertaken taking into account the whole spectrum of aspects of plasma modeling in tokamaks: MagnetoHydroDynamic (MHD) description of plasma interacting with the plasma facing components (pfc) of a tokamak structure. However, a cornerstone on developing such an approach is a correct, although simplified, description of the problem in purely EM terms.

The plasma radial current density distribution is considered to be equal to:

$$
J(r)=J_{0} e^{-a(r-R)^{2}}
$$

$\alpha$ being a shape factor ( $\alpha=0$ represents a uniform radial profile). 
Both cases (static and dynamic disruptions) can be dealt with by CARIDDI.

When a static disruption is considered, the user of the code must prepare a file of input for the code, containing information about the conductor of suitable section, and the decay of the current as a set of values of the plasma current at certain chosen instants. In order to model the gradually extinguishing plasma current, the user of the code must suppose the current density to be constant and the section of the conductor reduced at every time step of the resolution phase.

When a dynamic disruption is considered, CARIDDI enables the user to adequately model a vertical displacement of the plasma column by defining a set of axi-symmetric (toroidal) conductors one above the other. The current switches on in one conductor, only when it has switched off in the preceding one (this simulates a current moving up or down). The (rectangular) section of the various conductors can be changed. If plasma current decays (this is the case during VDEs), the cross-section of the (rectangular) conductors must be reduced by the user.

Results presented in the next paragraph are obtained by using the code CARIDDI as a modeling tool.

\section{Modeling hypotheses and results}

In a static disruption scenario, the plasma current decays to zero without any changes in the plasma ring position.

On the contrary, the plasma appears to have a vertical displacement and the plasma current may switch-off slowly during the displacement, or very rapidly at the end of it, when a dynamic disruption occurs.

Simulation of a downward VDE was carried out, the current density being considered constant. This mean that in this case the user of the CARIDDI code was not constrained to change the cross-section of the conductors carrying plasma current at every step of the resolution phase. The plasma cross-section was considered to have a rectangular shape.

According to studies carried out in the USA [6], the displacement of the plasma has been by an exponential movement:

$$
z=z_{e q}-z_{0} e^{\gamma t}
$$

in the ITER case the following data for constants being valid:

$$
\begin{aligned}
& Z_{\text {eq }}=1.30 \mathrm{~m} \text { equilibrium position } \\
& \mathrm{Z}_{0}=0.05 \mathrm{~m} \text { initial offset } \\
& \gamma=150 \mathrm{~s}^{-1} \text { growth rate. }
\end{aligned}
$$

Given a displacement time of $25 \mathrm{~ms}$, the growth rate is chosen to have about $2 \mathrm{~m}$ of total vertical motion, which is compatible with ITER's size. 
For the simulations, the following parameters have been taken:

- plasma current :

- plasma center position :

- elliptical plasma :

- rectangular plasma :
$25 \mathrm{MA}$

$\mathrm{r}=7.75 \mathrm{~m}$ in radial direction and

$\mathrm{Z}=1.3 \mathrm{~m}$ in vertical direction

minor semi-axis at equilibrium a $=3.0 \mathrm{~m}$

major semi-axis at equilibrium $b=4.7 \mathrm{~m}$

elongation $\mathrm{k}=\mathrm{a} / \mathrm{b}=1.6$

section $\mathrm{S}=44.27 \mathrm{~m}^{2}$

picked ( $\alpha=3$ ) or uniform ( $\alpha=0$ ) radial plasma current density profile

dimensions at eqiuilibrium : $\mathrm{D}_{\mathrm{x}}=6.00 \mathrm{~m}$; $\mathrm{D}_{\mathrm{y}}=9.40 \mathrm{~m} ; \mathrm{D}_{\mathrm{x}} / \mathrm{D}_{\mathrm{y}}=$ const. $=1.6$

section $\mathrm{S}=56.4 \mathrm{~m}^{2}$

flat radial plasma current density profile

- magnetic field at plasma center: $\quad 7.8 \mathrm{~T}$

In the modeling of the blanket module, four materials have been considered: materials 1 and 2 for the BP, and materials 3 and 4 for the FW (see figs. 1 and 2). Materia1s 1 and 2 (BP) are stainless steel, with a resistivity of $1.0 \times 10^{-6} \Omega \mathrm{m}$, whereas 3 and 4 present a higher resistivity, fixed to the value $1.5 \times 10^{-6} \Omega \mathrm{m}$, because the segmented curved configuration of the FW is taken into account.

The following reference disruption scenarios have been considered:

- model P1: linear current decay in $25 \mathrm{~ms}$ for an elliptical cross-section plasma (slow current quench).

- model P2: linear current decay in $25 \mathrm{~ms}$ for a rectangular cross-section plasma (slow current quench).

- model P3: vertical displacement over $2 \mathrm{~m}$ in $25 \mathrm{~ms}$ (pure VDE).

- model P4: vertica1 plasma motion of $2 \mathrm{~m}$ in $25 \mathrm{~ms}$ super-posed to a slow current quench (1 MA/ms).

- model P5: VDE (2 $\mathrm{m}$ in $25 \mathrm{~ms})$ followed by a fast current quench (5 MA/ms).

- model P6: linear current decay in $5 \mathrm{~ms}$ for an elliptical cross-section p1 asma (fast current quench).

- model P7 : linear current decay in $5 \mathrm{~ms}$ for a rectangular cross-section plasma (fast current quench).

Models P1, P2, P6 and P7 concern plasma disruptive instabilities known as static disruptions, whereas P3, P4 and P5 simulate plasma VDE accidental events known as dynamic disruptions. 

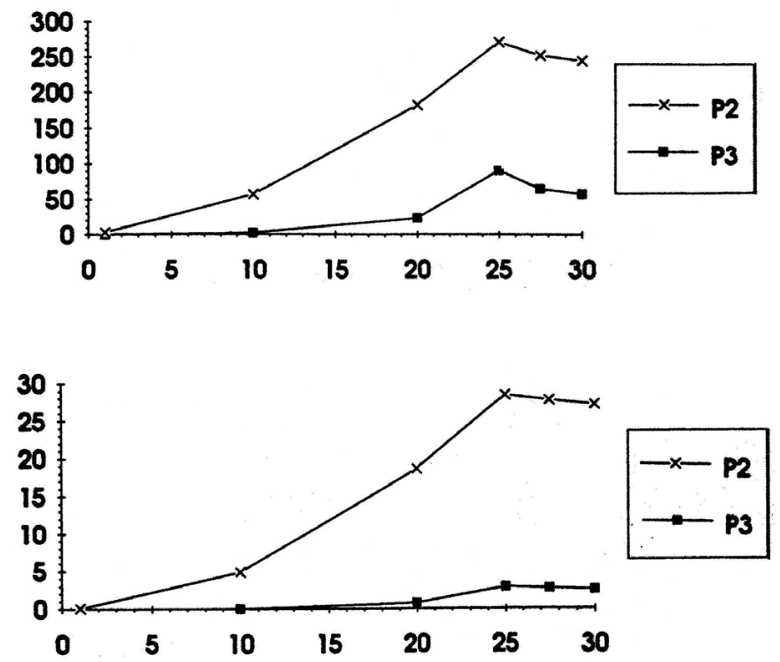

Figure 1: Graphic comparison of ohmic power measured in MW and magnetic energy measured in MJ for models P2 and P3 (time is measured in $\mathrm{ms}$ ).
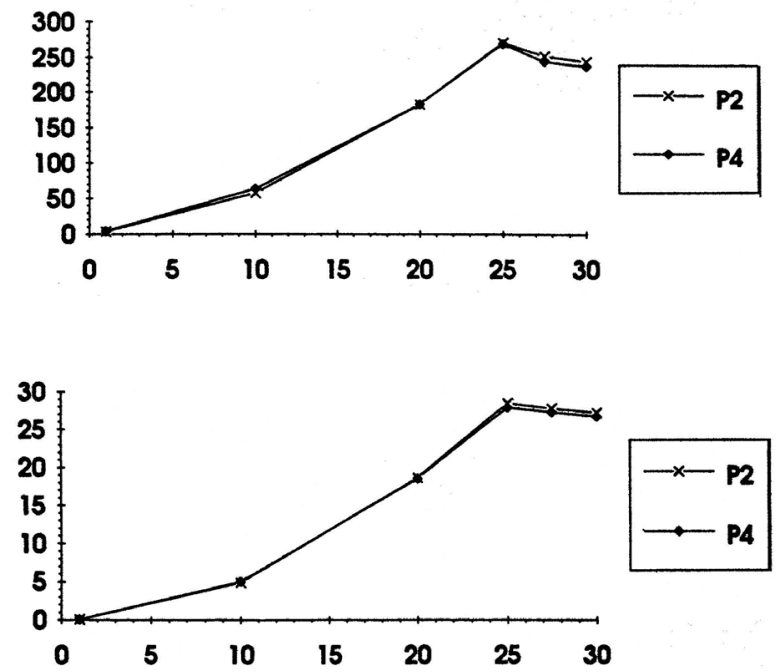

Figure 2: Graphic comparison of ohmic power measured in MW and magnetic energy measured in MJ for models P2 and P4 (time is measured in $\mathrm{ms}$ ). 

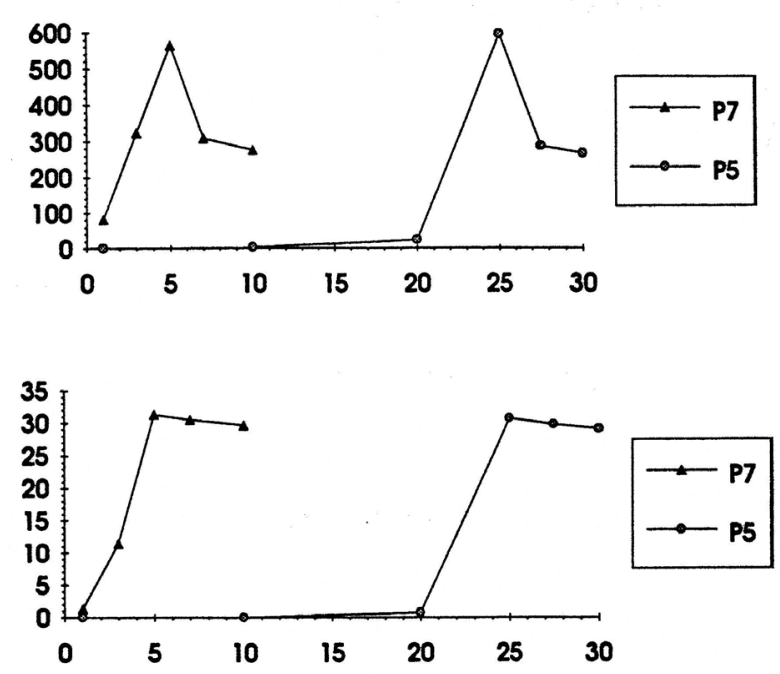

Figure 3: Graphic comparison of ohmic power measured in MW and magnetic energy measured in MJ for models P5 and P7 (time is measured in $\mathrm{ms}$ ).

Detailed results may be found in [7]. Some additional results are contained in figs. 1, 2 and 3 presenting ohmic power (P) dissipated and magnetic energy (W) stored in the blanket sector under study, due to eddy currents, for models P2, P3, P4, P5, P7.

\section{Conclusions}

The results clearly show that the effect of a pure VDE (model P3) is globally quite different from that of a pure (slow) current decay (model P2), having as a consequence the appearance of lower levels of ohmic power and magnetic energy (fig. 4 of [7]). On the other hand, if models P2 and P4 are compared, it is observed, once again, that as far as a blanket is concerned, vertical displacement of the plasma does not produce essentially new effects, due to the fact that the slow current quench accidental event is pre-dominant (fig. 5 of [7]). When the current quench rate is increased, as is done in models P5 and P7, higher levels of ohmic power are observed (fig. 6 of [7]). However, a shift of the $\mathrm{P}$ and $\mathrm{W}$ patterns is noticed when current decays at the end of VDEs (as it is apparent in model P5). This is an indication element, once more, showing that current quench is pre-dominant in these accidental scenarios too. Furthermore, higher values of $\mathrm{P}$, when eddy currents increase, is an indication element showing that, in blankets, which are continuous, as far as it concerns the toroidal direction, resistive effects are much more important than the inductive ones. 


\section{Acknowledgements}

Mrs Sophia Fantechi, specialist in the plasma aspects of the project, Mr Yves Robert Crutzen, supervisor of the whole project and specialist in the mechanical aspects of the project, Mrs Areti Malkogianni and Mrs Magdalene Chatzikosta for their help in typing, are acknowledged.

\section{References}

[1] Belyakov V.A. and The Russian Federation Participant Team, Russian Federation contribution to the ITER project, Fusion Engineering and Design, Volume 81, Issues 1-7, February 2006, 79-86.

[2] Mirnov S.V., Evtikhin V.A., The tests of liquid metals (Ga, Li) as plasma facing components in T-3M and T-11M tokamaks, Fusion Engineering and Design, Volume 81, Issues 1-7, February 2006,113-119.

[3] Rubel M.J., Fortuna E., Kreter A., Wessel E., Philipps V., Kurzydłowski K.J., Overview of comprehensive characterisation of erosion zones on plasma facing components, Fusion Engineering and Design, Volume 81, Issues 1-7, February 2006, 211-219.

[4] A. Grosman and Tore Supra team, High heat flux actively cooled plasma facing components development, realisation and first results in Tore Supra, Fusion Engineering and Design, Volume 74, Issues 1-4, November 2005, 49-57.

[5] Darke A.C., Hayward R.J., Counsell G.F., Hawkins K., The MAST improved divertor Fusion Engineering and Design, Volumes 75-79, November 2005, 285-289.

[6] Loustenau D. C., Williamson D. E., Vacuum Vessel Design, presented at ITER Technical Meeting, Garching (D), 1993.

[7] Sakellaris J., Simplified modeling of tokamak plasmas in a computational electromagnetic environment, Energy and the environment, Editors: C. A. Brebbia and I. Sakellaris, WIT PRESS, 307-317, May 2003, ISBN 1 - 85312 $-970-4$ 